\title{
Endovascular stroke intervention in young patients with large vessel occlusions
}

\author{
Nohra Chalouhi, M.D., ${ }^{1}$ Stavropoula TJoumakaris, M.D., ${ }^{1}$ Robert M. Starke, M.D., ${ }^{2}$ \\ David Hasan, M.D., ${ }^{3}$ Nimrita Sidhu, B.S., 1 Saurabh Singhal, M.D., ${ }^{1}$ \\ Shannon Hann, M.D., ${ }^{1}$ L. Fernando Gonzalez, M.D., ${ }^{1}$ Robert Rosenwasser, M.D., 1 \\ and Pascal Jabbour, M.D. ${ }^{1}$ \\ ${ }^{1}$ Department of Neurosurgery, Thomas Jefferson University and Jefferson Hospital for Neuroscience, \\ Philadelphia, Pennsylvania; ${ }^{2}$ Department of Neurosurgery, University of Virginia School of Medicine, \\ Charlottesville, Virginia; and ${ }^{3}$ Department of Neurosurgery, Carver College of Medicine, University of Iowa, \\ Iowa City, Iowa
}

\begin{abstract}
Object. Endovascular therapy has become a widely used method for achieving arterial recanalization in patients who are ineligible for intravenous thrombolysis or those in whom it is unsuccessful. Young stroke patients with large vessel occlusions may particularly benefit from endovascular intervention. This study aims to assess the authors' experience with the use of modern endovascular techniques to treat young patients ( $\leq 55$ years old) with acute ischemic stroke and large vessel occlusions.

Methods. Young patients ( $\leq 55$ years old) undergoing endovascular intervention for acute ischemic stroke at the authors' institution were identified from a prospectively maintained database. Only those patients with a confirmed large vessel occlusion were included. Modified Rankin Scale (mRS) scores were determined at 90 days during a follow-up visit. A multivariate analysis was performed to determine predictors of outcome (mRS score 0-2).

Results. A total of 45 patients met the inclusion criteria. The mean age of the patients in this series was $45 \pm$ 9.6 years. The mean admission NIH Stroke Scale score was $14.1 \pm 5$ (median 13.5). Mechanical thrombectomy was performed using the Solitaire FR device in 13 (29\%) patients and the Merci/Penumbra systems in 32 (71\%) patients. The rate of successful recanalization (Thrombolysis In Myocardial Infarction [TIMI] scale Grade II-III) was $93 \%(42 / 45)$. Only 1 patient $(2.2 \%)$ had a symptomatic intracranial hemorrhage following intervention. One patient $(2.2 \%)$ sustained a vessel perforation intraoperatively. The rate of 90-day favorable outcome (mRS score 0-2) was $77.5 \%$ and the rate of 90 -day satisfactory outcome (mRS score $0-3$ ) was $90 \%$. The 90 -day mortality rate was $7.5 \%$. In multivariate analysis, postprocedure TIMI grade was the only statistically significant independent predictor of 90-day outcome (OR 3.3, 95\% CI 1.01-1.19; $\mathrm{p}=0.05$ ).

Conclusions. The results of this study demonstrate that endovascular therapy provides remarkably high rates of arterial recanalization and favorable outcomes in young patients with acute ischemic stroke and large vessel occlusions. These findings support aggressive interventional strategies in these patients. Randomized, controlled trials reflecting modern acute ischemic stroke treatment will be needed to confirm the findings of this study. (http://thejns.org/doi/abs/10.3171/2013.9.FOCUS13398)
\end{abstract}

\section{KeY Words • endovascular therapy - large vessel occlusion • acute ischemic stroke}

$\mathrm{S}$ TROKE is the leading cause of adult disability and the third-leading cause of death in the US. ${ }^{17}$ Intravenous recombinant tissue plasminogen activator (rtPA) is the only therapy approved by the FDA for treatment of acute ischemic stroke. ${ }^{20}$ Currently, the indication for intravenous rtPA extends to 4.5 hours after symptom onset in eligible stroke patients lacking contraindications. ${ }^{11}$ In patients who are ineligible for intravenous rtPA, or those in whom it is unsuccessful, endovascular therapy has become a widely used method for achieving arterial recanalization. $7,10,19,22$

\footnotetext{
Abbreviations used in this paper: IMS III = Interventional Management of Stroke III; mRS = modified Rankin Scale; NIHSS = NIH Stroke Scale; rtPA = recombinant tissue plasminogen activator; TIMI = Thrombolysis In Myocardial Infarction .
}

Endovascular stroke intervention is particularly indicated for patients with large-vessel occlusions, ${ }^{14}$ for whom revascularization rates with intravenous rtPA alone are suboptimal, and range from $10 \%$ in internal carotid artery occlusion to less than $30 \%$ for middle cerebral artery occlusion. ${ }^{23}$ Indeed, subgroup analysis of the recently published Interventional Management of Stroke III (IMS III) trial showed a significant benefit with intraarterial therapy in patients with large vessel occlusions, ${ }^{3}$ suggesting that endovascular interventions are beneficial when used in the appropriate setting. Along these lines, we hypothesize that young patients with acute ischemic stroke and large vessel occlusions fare particularly well with endovascular therapy, which could support a strategy of aggressive intervention in these patients. However, data on the safety and efficacy of endovascular stroke intervention in this subset of patients are lacking. In the present study, we re- 
view our experience with the use of modern endovascular techniques to treat young patients ( $\leq 55$ years) with acute ischemic stroke and large vessel occlusions.

\section{Methods}

\section{Study Population}

The study protocol was approved by the Thomas Jefferson University Institutional Review Board. Young patients ( $\leq 55$ years old) who underwent endovascular intervention for acute ischemic stroke at Jefferson Hospital for Neuroscience between January 2008 and March 2013 were identified from a prospectively maintained database. Only those with a confirmed large vessel occlusion (vertebral, basilar, internal carotid, middle and anterior cerebral arteries) were included. A total of 45 patients met the inclusion criteria and constituted our study population.

\section{Patient Selection}

Patients with suspected acute stroke are admitted from the Thomas Jefferson University Hospital emergency department, transferred from affiliated community hospitals, or directly accepted by the attending physician on telestroke call. ${ }^{5}$ Under our current institutional protocol, all patients suspected of acute ischemic stroke undergo immediate noncontrast CT, CT angiography, and CT perfusion upon referral to our center. Patients with an NIH Stroke Scale (NIHSS) score greater than or equal to 5-8 and evidence of a large penumbra on CT perfusion ${ }^{2}$ (decreased cerebral blood flow, preserved cerebral blood volume, and increased mean transit time in more than $30 \%-$ $50 \%$ of the affected territory), in conjunction with visible main arterial thrombus on CT angiography, are selected to undergo an intraarterial intervention. Contraindications to intervention include improving neurological status with low NIHSS score, extreme old age, and multiple medical comorbidities such as preexisting elevated international normalized ratio. Previous administration of intravenous rtPA does not usually preclude an intraarterial intervention in patients with worsening neurological status.

\section{Endovascular Technique}

Procedures are performed under general endotracheal anesthesia and maintenance of systolic blood pressure between 160 and $200 \mathrm{~mm} \mathrm{Hg}(<185 \mathrm{~mm} \mathrm{Hg}$ if intravenous rtPA was given). Heparin is administered to maintain the activated clotting time between 200 and 300 seconds throughout the procedure. Mechanical thrombectomy is performed with the Penumbra System (Penumbra Inc.), the Merci Retriever (Concentric Medical), or more recently with the Solitaire FR device (eV3). Intraarterial rtPA and Abciximab (ReoPro, Eli Lilly) are used in some patients as an adjunct to mechanical devices. Postoperatively, all patients are transferred to the neurointensive care unit, after which a noncontrast head CT is routinely obtained to diagnose hemorrhagic complications.

\section{Data Collection}

The patient's neurological status was assessed using the NIHSS on admission. The extent of recanalization was classified according to the Thrombolysis In Myocardial Infarction (TIMI) grading system as follows: $0=$ no recanalization, I = minimal recanalization, $\mathrm{II}=$ partial recanalization, and III = complete recanalization. Safety outcome was assessed by intracranial hemorrhage postintervention, device-induced vessel damage and further propagation of thrombus, and mortality rate at 90 days. Intracranial hemorrhage was classified as symptomatic when it was associated with worsening of NIHSS score by 4 or more points, per the classification of the EuropeanAustralasian Acute Stroke Study (ECASS). ${ }^{12}$ Clinical outcomes were determined at 90 days during a follow-up visit and categorized according to the modified Rankin Scale (mRS) score.

\section{Statistical Analysis}

Data are presented as mean and range for continuous variables and as frequency for categorical variables. Statistical analysis of categorical variables was performed using chi-square and Fisher's exact tests, as appropriate. Comparison of means was performed using the Student t-test. Interaction and confounding were assessed through stratification and relevant expansion covariates. A multivariable logistic regression analysis was conducted to determine predictors of favorable outcomes (mRS score $0-2)$. Factors predictive in univariate analysis $(\mathrm{p}<0.15)^{1}$ were entered into a stepwise backward multivariate logistic regression analysis; $\mathrm{p}$ values $\leq 0.05$ were considered statistically significant. Statistical analysis was performed using Stata statistical software (version 10.0, StataCorp LP).

\section{Results}

Twenty-five patients (56\%) were female and 20 (44\%) were male. The mean age of the patients in the series was $45 \pm 9.6$ years. Ten patients $(22 \%)$ were younger than 35 years, $7(16 \%)$ were between 36 and 45 years, and 28 (62\%) were between 46 and 55 years. The mean admission NIHSS score was $14.1 \pm 5$ (median 13.5).

Intravenous rtPA was administered before initiation of intraarterial therapy in 23 patients (51\%). Thirty-three patients $(73 \%)$ were selected based on CT perfusion criteria. The mean duration from symptom onset to femoral artery puncture was $6.7 \pm 3.6$ hours. Arterial occlusion sites were as follows: middle cerebral artery in 29 $(64 \%)$, internal carotid artery occlusion in $3(7 \%)$, internal carotid artery $\mathrm{T}$ occlusion in $4(9 \%)$, and vertebrobasilar in $9(20 \%)$. Mechanical thrombectomy was performed using the Solitaire FR device in 13 patients $(29 \%)$ and the Merci/Penumbra systems in $32(71 \%)$ patients.

The rate of successful recanalization (TIMI Grade II-III) was $93 \%(42 / 45)$. The TIMI grades were 0 in 1 patient $(2 \%)$, I in 2 patients (4\%), II in 7 patients $(16 \%)$, and III in 35 patients $(78 \%)$. Only 1 patient $(2 \%)$ had a symptomatic intracranial hemorrhage following intervention. One patient (2\%) sustained a vessel perforation intraoperatively. Forty of 45 patients had available clinical follow-up. The rate of 90-day favorable outcome (mRS score $0-2)$ was $77.5 \%(31 / 40)$, and the rate of 90-day satisfactory outcome (mRS score 0-3) was $90 \%$ (36/40). The 90-day mortality rate was $7.5 \%$ (3/40). 
The rate of 90-day favorable outcome (mRS score 0-2) was $89 \%(8 / 9)$ for patients younger than 35 years, $67 \%(4 / 6)$ for those between 36 and 45 years, and 76\% $(19 / 25)$ for those between 46 and 55 years $(p=0.3)$. Favorable outcomes were noted in $85 \%(11 / 13)$ of patients treated with Solitaire FR device and 74\% (20/27) of those treated with Merci/Penumbra devices $(p=0.4)$. The following factors were tested as predictors of 90-day outcome: age, sex, NIHSS score, vessel location, use of CT perfusion, use of intravenous rtPA, time from symptom onset to femoral artery puncture, type of device, and postprocedure TIMI grade. In univariate analysis, increasing postprocedure TIMI grade $(\mathrm{p}=0.05)$ predicted favorable outcome. In multivariate analysis, postprocedure TIMI grade was the only statistically significant independent predictor of 90-day outcome (OR 3.3, 95\% CI 1.01-1.19; $\mathrm{p}=0.05)$.

\section{Discussion}

Because of their long life expectancy, young patients account for up to $20 \%$ of potential life-years lost due to stroke. ${ }^{4}$ Thus, successful management of acute ischemic stroke in young patients has important public health implications. These implications are even more important in young patients with large vessel occlusions given the devastating natural history of such strokes. The Prolyse in Acute Cerebral Thromboembolism II (PROACT II) study showed a $75 \%$ poor outcome rate in patients with confirmed large-vessel occlusions when intraarterial therapy was not undertaken. ${ }^{8}$ Likewise, preliminary results from the FIRST trial, a prospective, multicenter, natural history study of patients eligible for but untreated by mechanical thrombectomy from a large vessel occlusion, revealed that only $20 \%$ of patients achieved a good 90 -day outcome and as many as $40.7 \%$ died. ${ }^{13}$ Thus, endovascular intervention in young patients with large vessel occlusions has the potential to provide significant clinical and public health benefits. Yet, data on the value of endovascular stroke therapy in this subgroup have been surprisingly scarce. We are aware of only 1 study that specifically assessed the safety and efficacy of endovascular stroke intervention in young patients. In this small series, Mocco et al.${ }^{18}$ reported on 7 young patients ( $\leq 35$ years old) who underwent endovascular interventions for thromboembolic stroke. There were no deaths in their series, and all 7 patients achieved functional independence at follow-up. The authors concluded that endovascular intervention is relatively safe in young patients and may provide a potential benefit in outcome.

Publication of the IMS III, Mechanical Retrieval and Recanalization of Stroke Clots Using Embolectomy (MR RESCUE), and Intra-Arterial versus Systemic Thrombolysis for Acute Ischemic Stroke (SYNTHESIS) results, all of which failed to show a significant benefit with endovascular therapy over intravenous rtPA alone in acute ischemic stroke, has generated negative press about the value of interventional stroke intervention. ${ }^{3,6,15}$ Although the trials failed to identify large vessel occlusions before enrollment and did not meet current revascularization standards (due to use of first-generation thrombectomy devices), they have prompted an emphasis on the identification of subgroups of patients in whom intervention might be beneficial. Clearly, in light of our results, young patients with large vessel occlusions may be one such group. Indeed, in the present study, $77.5 \%$ of patients achieved an excellent outcome (mRS score 0-2) at follow-up and up to $90 \%$ achieved a good outcome (mRS score $0-3$ ). In comparison, only $53 \%$ of patients suffering acute ischemic stroke at 45 years old or younger in a community study experienced a good recovery. ${ }^{16}$ Although our study did not formally include a control group treated with intravenous rtPA alone, our favorable results strongly support a strategy of aggressive intervention in young patients with acute ischemic stroke and large vessel occlusions. This is further supported by the poor outcomes associated with noninterventional strategies in large-vessel occlusion strokes, as discussed above. Moreover, the rates of favorable outcomes in our study are well above those in published trials on intraarterial therapy. For example, the rate of favorable outcomes was only $40.8 \%$ in the IMS III trial ${ }^{3}$ and $30.4 \%$ in the SYNTHESIS Trial. ${ }^{6}$

We experienced a recanalization rate as high as $93 \%$. This high recanalization rate highlights the value of intervention in young patients with large vessel occlusions. In fact, young patients have a less tortuous and atherosclerotic arterial anatomy with more robust vascular walls, which facilitates endovascular intervention and potentially reduces procedural complications. As such, there was only 1 instance of vessel rupture $(2.2 \%)$ and only 1 clinically symptomatic intracerebral hemorrhage postprocedurally $(2.2 \%)$. To put these numbers into perspective, in the recent IMS III trial, the recanalization rate was only $40 \%$ and the intracerebral hemorrhage rate was $6.2 \%$. In the large Swiss series by Galimanis et al., ${ }^{9}$ successful recanalization was achieved in $70.3 \%$ and symptomatic intracerebral hemorrhage occurred in 5.5\%. Again, our results emphasize the safety and efficacy of intraarterial therapy in promoting favorable outcomes in young patients with acute ischemic stroke.

The multivariate analysis identified successful recanalization as a predictor of favorable outcome in young patients with acute ischemic stroke. This finding highlights the importance of achieving arterial recanalization in a timely manner in young patients with large vessel occlusions. Although our study was underpowered to detect differences in subgroups of patients, we observed some interesting trends that are worth mentioning. First, excellent outcomes (mRS score 0-2) occurred in $74 \%$ of patients older than 35 years compared with as many as $89 \%$ of patients younger than 35 years. This interesting finding supports a particularly aggressive interventional strategy in very young acute ischemic stroke patients. Second, favorable outcomes were noted in $85 \%$ of patients treated with Solitaire FR device versus $74 \%$ of those treated with Merci/Penumbra devices. Although this difference did not reach statistical significance, the finding appears to replicate the results of the Solitaire With the Intention For Thrombectomy (SWIFT) trial in which higher recanalization rates (61\% vs 24\%) and better neurological outcomes (58\% vs 33\%) were found with the Solitaire FR device compared with the Merci device. ${ }^{21}$ However, larger studies are needed to confirm these trends.

The limitations of our study are related to its retro- 
spective design and the absence of a control group treated with intravenous rtPA alone. There is currently no standard definition for young age in acute ischemic stroke. We chose a cutoff of 55 years to provide a sizable sample of patients, taking into consideration the fact that individuals younger than 55 years of age usually remain healthy. A more conservative approach would have been to select a cutoff of 35 or 45 years of age. The time interval from symptom onset to femoral puncture was rather long, but this is expected given our reliance on CT perfusion and the fact that most patients are transferred from affiliated community hospitals.

\section{Conclusions}

The results of this study suggest that endovascular therapy provides remarkably high rates of arterial recanalization and favorable outcomes in young patients with acute ischemic stroke and large vessel occlusions. These findings support aggressive interventional strategies in these patients. Randomized, controlled trials reflecting modern acute ischemic stroke treatment will be needed to confirm the findings of this study.

\section{Disclosure}

Dr. Jabbour serves as a consultant to Covidien and CNV.

Author contributions to the study and manuscript preparation include the following. Conception and design: Jabbour, Tjoumakaris, Starke, Gonzalez, Rosenwasser. Acquisition of data: Chalouhi, Sidhu, Singhal, Hann. Analysis and interpretation of data: Jabbour, Chalouhi, Tjoumakaris, Starke, Hasan, Sidhu, Singhal, Hann. Drafting the article: Jabbour, Chalouhi, Tjoumakaris, Hasan, Gonzalez. Critically revising the article: Jabbour, Chalouhi, Tjoumakaris, Starke, Hasan, Sidhu, Hann, Gonzalez, Rosenwasser. Reviewed submitted version of manuscript: all authors. Approved the final version of the manuscript on behalf of all authors: Jabbour. Statistical analysis: Starke. Administrative/technical/material support: Jabbour, Chalouhi. Study supervision: Jabbour, Chalouhi.

\section{References}

1. Altman DG: Practical Statistics for Medical Research. Boca Raton, FL: CRC Press, 1999

2. Amenta PS, Ali MS, Dumont AS, Gonzalez LF, Tjoumakaris SI, Hasan D, et al: Computed tomography perfusion-based selection of patients for endovascular recanalization. Neurosurg Focus 30(6):E6, 2011

3. Broderick JP, Palesch YY, Demchuk AM, Yeatts SD, Khatri P, Hill MD, et al: Endovascular therapy after intravenous t-PA versus t-PA alone for stroke. N Engl J Med 368:893-903, 2013

4. Carolei A, Marini C, Di Napoli M, Di Gianfilippo G, Santalucia $\mathrm{P}$, Baldassarre $\mathrm{M}$, et al: High stroke incidence in the prospective community-based L'Aquila registry (1994-1998). First year's results. Stroke 28:2500-2506, 1997

5. Chalouhi N, Dressler JA, Kunkel ES, Dalyai R, Jabbour P, Gonzalez LF, et al: Intravenous tissue plasminogen activator administration in community hospitals facilitated by telestroke service. Neurosurgery 73:667-672, 2013

6. Ciccone A, Valvassori L, Nichelatti M, Sgoifo A, Ponzio M, Sterzi R, et al: Endovascular treatment for acute ischemic stroke. N Engl J Med 368:904-913, 2013

7. Day JS, Hurley MC, Chmayssani M, Rahme RJ, Alberts MJ, Bernstein RA, et al: Endovascular stroke therapy: a singlecenter retrospective review. Neurosurg Focus 30(6):E10, 2011

8. Furlan A, Higashida R, Wechsler L, Gent M, Rowley H, Kase $\mathrm{C}$, et al: Intra-arterial prourokinase for acute ischemic stroke.
The PROACT II study: a randomized controlled trial. JAMA 282:2003-2011, 1999

9. Galimanis A, Jung S, Mono ML, Fischer U, Findling O, Weck A, et al: Endovascular therapy of 623 patients with anterior circulation stroke. Stroke 43:1052-1057, 2012

10. Ghobrial GM, Chalouhi N, Rivers L, Witte S, Davanzo J, Dalyai R, et al: Multimodal endovascular management of acute ischemic stroke in patients over 75 years old is safe and effective. J Neurointerv Surg 5 Suppl 1:i33-i37, 2013

11. Hacke W, Kaste M, Bluhmki E, Brozman M, Dávalos A, Guidetti D, et al: Thrombolysis with alteplase 3 to 4.5 hours after acute ischemic stroke. N Engl J Med 359:1317-1329, 2008

12. Hacke W, Kaste M, Fieschi C, von Kummer R, Davalos A, Meier D, et al: Randomised double-blind placebo-controlled trial of thrombolytic therapy with intravenous alteplase in acute ischaemic stroke (ECASS II). Lancet 352:1245-1251, 1998

13. Janardhan V, Gianatasio RM, Chen SH, Bhuva P, Murray MM, Santos P, et al: Abstract 194: Preliminary results from the FIRST trial: natural history of acute stroke from large vessel occlusion. Stroke 44:A194, 2013 (Abstract)

14. Khalessi AA, Fargen KM, Lavine S, Mocco J: Commentary: Societal statement on recent acute stroke intervention trials: results and implications. Neurosurgery 73:E375-E379, 2013

15. Kidwell CS, Jahan R, Gornbein J, Alger JR, Nenov V, Ajani Z, et al: A trial of imaging selection and endovascular treatment for ischemic stroke. N Engl J Med 368:914-923, 2013

16. Marini C, Totaro R, De Santis F, Ciancarelli I, Baldassarre M, Carolei A: Stroke in young adults in the community-based L'Aquila registry: incidence and prognosis. Stroke 32:52-56, 2001

17. Meyers PM, Schumacher HC, Connolly ES Jr, Heyer EJ, Gray WA, Higashida RT: Current status of endovascular stroke treatment. Circulation 123:2591-2601, 2011

18. Mocco J, Tawk RG, Jahromi BS, Samuelson RM, Siddiqui AH, Hopkins LN, et al: Endovascular intervention for acute thromboembolic stroke in young patients: an ideal population for aggressive intervention? Clinical article. J Neurosurg 110: 30-34, 2009

19. Mokin M, Dumont TM, Veznedaroglu E, Binning MJ, Liebman KM, Fessler RD II, et al: Solitaire Flow Restoration thrombectomy for acute ischemic stroke: retrospective multicenter analysis of early postmarket experience after FDA approval. Neurosurgery 73:19-26, 2013

20. National Institute of Neurological Disorders and Stroke rt-PA Stroke Study Group: Tissue plasminogen activator for acute ischemic stroke. N Engl J Med 333:1581-1588, 1995

21. Saver JL, Jahan R, Levy EI, Jovin TG, Baxter B, Nogueira $\mathrm{RG}$, et al: Solitaire flow restoration device versus the Merci Retriever in patients with acute ischaemic stroke (SWIFT): a randomised, parallel-group, non-inferiority trial. Lancet 380: 1241-1249, 2012

22. Starke RM, Komotar RJ, Connolly ES: Mechanical clot retrieval in the treatment of acute ischemic stroke. Neurosurgery 72:N19-N21, 2013

23. Wolpert SM, Bruckmann H, Greenlee R, Wechsler L, Pessin MS, del Zoppo GJ: Neuroradiologic evaluation of patients with acute stroke treated with recombinant tissue plasminogen activator. AJNR Am J Neuroradiol 14:3-13, 1993

\footnotetext{
Manuscript submitted September 10, 2013.
}

Accepted September 17, 2013.

Please include this information when citing this paper: DOI: 10.3171/2013.9.FOCUS13398.

Address correspondence to: Pascal M. Jabbour, M.D., Division of Neurovascular Surgery and Endovascular Neurosurgery, Department of Neurological Surgery, Thomas Jefferson University Hospital, 901 Walnut St., 3rd Floor, Philadelphia, PA 19107. email: pascal.jabbour@jefferson.edu. 